\title{
TOWARD A BETTER ASSESSMENT OF STUDENTS OUTCOMES USING A SIX-STEPS METHOD AND A CLASS DIAGRAM
}

\author{
= SAMIRKAOUCETTA \\ SAMIR K. BOUCETTA \\ IT Department, Faculty of Computing and Information Technology in Rabigh, \\ King Abdulaziz University, Saudi Arabia \\ Emails: sBoucetta@kau.edu.sa
}

\begin{abstract}
Revised September 2017
ABSTRACT. The achievement of the ABET Students Outcomes (SOs) aims to ensure the good preparation of the students to the workplace. The evaluation of the SOs attainment faces many problems including the partial achievement of SO and the appropriate mapping of the assessment tools to SOs. Furthermore, the complexity and heaviness of the evaluation process require a 2-4 years improvement cycle to avoid faculty overloading. In this work, initially, a class diagram is developed which shows the classes of the system, their attributes, operations and the relationships among objects. Further, we propose a six-steps methodology that aims to better undertake the assessment of the SOs for an IT program. The first step proceeds to the preparation of an articulation matrix that maps the IT program courses to the SOs. The second step decomposes each SO into its Elementary contents (SOES) in order to target each SOE separately and contribute to the full achievement of the SO. The detailed articulation matrix is prepared in the third step to include the new list of SOEs. While undertaking the semester-based assessments, the fourth step consists in mapping the assessment tools and questions to the appropriate SOEs using the Bloom's measurable verbs. At the end of the semester, the fifth step is undertaken to input the assessment data for each SOE. The final sixth step proceeds to the analysis of the assessment data to outline important results at both course and program levels. This work is undertaken at the Faculty of Computing and Information Technology in Rabigh (FCITR), King Abdulaziz University, and will be soon supported by a web-based system that helps in the implementation of the six-steps methodology, which will considerably shorten the improvement cycle.
\end{abstract}

Keywords: Student Outcomes Assessment, Six-Steps Method, Class Diagram, Bloom's taxonomy, Bloom's measurable verbs

1. Introduction: One of the important ABET general criteria required for computing programs accreditation is the third criterion "Student Outcomes" (SOs). These outcomes prepare graduates to attain the program educational objectives [5]. The IT SOs include the nine SOs (a to i) common to all computing programs plus five IT specific SOs ( $\mathrm{j}$ to $\mathrm{n}$ ) as shown in the Figure 1. The implementation of these SOs is undertaken over the different courses of the IT program. The articulation matrix as shown in the Figure 2 shows the SOs that are targeted by each IT course of the IT program at the Faculty of Computing and Information Technology in Rabigh (FCITR).

As stated by ABET, an assessment is one or more processes that identify, collect, and prepare data to evaluate the attainment of SOs [5]. Effective assessment uses relevant direct, indirect, quantitative and qualitative measures as appropriate to the outcome being measured. The evaluation consists in the interpretation of the data and evidence accumulated through assessment processes. Evaluation determines the extent to which student outcomes are being attained [5].

Multiple types of direct and indirect assessments are used to evaluate the extent to which the SOs are being attained. Direct assessments provide for the direct examination of student knowledge or skills and are much more difficult to achieve [11]. Direct methods include observing students and examining their work. 
Indirect methods are much easier to design and implement as they involve self-reporting about the extent of the learning experience. Although, indirect methods are useful, they rely mostly on opinion or selfreporting [8] and may be affected by personal issues such as motivation. In order to have a most effective assessment process, this work makes use of both direct and indirect methods including multiple types of assessments as shown in the Figure 3. Each assessment - either direct or indirect- is composed of a set of questions. While preparing an assessment, its questions should be mapped to the appropriate SO. A bad mapping of the questions to the appropriate SO yield to flawed assessment data and compromises the reliability and the effectiveness of the evaluation process.

However, each SO, as stated by the ABET, addresses a set of knowledge and skills. For example, $\mathrm{SO}(\mathrm{I})$ "An ability to use current techniques, skills, and tools necessary for computing practice" addresses the use of current (i) techniques (ii) skills and (iii) tools necessary for computing practice. The implementation of the SO may be partially accomplished if one of the sub contents of the SO, namely, the techniques, skills and tools were not addressed by the assessments. Assessing the SO as "all-in-one" may be inaccurate. Hence, the SO should be studied carefully and decomposed into more elementary contents that should be addressed individually for better implementation of the SO.

\section{IT STUDENTS OUTCOMES}

The program must enable students to attain, by the time of graduation:

(a) An ability to apply knowledge of computing and mathematics appropriate to the program's student outcomes and to the discipline;

(b) An ability to analyze a problem, and identify and define the computing requirements appropriate to its solution;

(c) An ability to design, implement, and evaluate a computer-based system, process, component, or program to meet desired needs;

(d) An ability to function effectively on teams to accomplish a common goal;

(e) An understanding of professional, ethical, legal, security and social issues and responsibilities;

(f) An ability to communicate effectively with a range of audiences;

(g) An ability to analyze the local and global impact of computing on individuals, organizations, and society;

(h) Recognition of the need for an ability to engage in continuing professional development; (i) An ability to use current techniques, skills, and tools necessary for computing practice.

(j) An ability to use and apply current technical concepts and practices in the core information technologies. [IT]

(k) An ability to identify and analyze user needs and take them into account in the selection, creation, evaluation and administration of computer-based systems. [IT]

(l) An ability to effectively integrate IT-based solutions into the user environment. [IT]

(m) An understanding of best practices and standards and their application. [IT]

(n) An ability to assist in the creation of an effective project plan. [IT]

Figure 1 - The ABET Student Outcomes for an IT program

\begin{tabular}{|c|c|c|c|c|c|c|c|c|c|c|c|c|c|c|c|}
\hline \multirow{2}{*}{ Subjects } & \multirow[b]{2}{*}{ Code } & \multicolumn{14}{|c|}{ IT Student Outcomes } \\
\hline & & A & $\mathrm{B}$ & $\mathrm{C}$ & $\mathrm{D}$ & $\mathrm{E}$ & $\mathrm{F}$ & $\mathrm{G}$ & $\mathrm{H}$ & I & $\mathrm{J}$ & $\mathrm{K}$ & $\mathrm{L}$ & M & $\mathrm{N}$ \\
\hline Fundamentals of Computing & COIT 260 & $\sqrt{ }$ & & & & & & & & $\sqrt{ }$ & & & & & \\
\hline Human Computer Interaction & COIT 283 & & & $\sqrt{ }$ & & & & & & $\sqrt{ }$ & & $\sqrt{ }$ & & $\sqrt{ }$ & \\
\hline Computer Networks & COIT 374 & $\sqrt{ }$ & & & & $\sqrt{ }$ & & & & & & & & $\sqrt{ }$ & \\
\hline Object-Oriented Analysis and Design & COIT 415 & & $\sqrt{ }$ & $\sqrt{ }$ & & & & & & $\sqrt{ }$ & & $\sqrt{ }$ & & & \\
\hline IT Fundamentals & COIT 321 & $\sqrt{ }$ & & & & & & & & & $\sqrt{ }$ & & $\sqrt{ }$ & & \\
\hline Information Security & COIT 324 & $\sqrt{ }$ & $\sqrt{ }$ & & & $\sqrt{ }$ & & & & & & & & & \\
\hline Database Administration & COIT 342 & & & & & $\sqrt{ }$ & & & & & $\sqrt{ }$ & & & $\sqrt{ }$ & \\
\hline Integrative Programming \& Technologies & COIT 403 & $\sqrt{ }$ & & $\sqrt{ }$ & & & & & & & & & $\sqrt{ }$ & & \\
\hline System Administration and Maintenance & COIT 411 & & & $\sqrt{ }$ & & & & $\sqrt{ }$ & & $\sqrt{ }$ & & & & & \\
\hline System Integration and Architecture & COIT 412 & & $\sqrt{ }$ & & & $\sqrt{ }$ & & & & & & & $\sqrt{ }$ & & $\sqrt{ }$ \\
\hline Web Systems and Technologies & COIT 463 & & $\sqrt{ }$ & $\sqrt{ }$ & $\sqrt{ }$ & & & & & $\sqrt{ }$ & & & & & \\
\hline Multimedia Technologies & COIT 466 & & $\sqrt{ }$ & $\sqrt{ }$ & & & & & & $\sqrt{ }$ & & & & & \\
\hline Senior Project 1 & COIT 498 & $\sqrt{ }$ & $\sqrt{ }$ & $\sqrt{ }$ & $\sqrt{ }$ & & $\sqrt{ }$ & & $\sqrt{ }$ & $\sqrt{ }$ & & $\sqrt{ }$ & & & $\sqrt{ }$ \\
\hline Senior Project 2 & COIT 499 & $\sqrt{ }$ & & $\sqrt{ }$ & $\sqrt{ }$ & & $\sqrt{ }$ & $\sqrt{ }$ & $\sqrt{ }$ & $\sqrt{ }$ & & & $\sqrt{ }$ & & $\sqrt{ }$ \\
\hline
\end{tabular}

Figure 2-The Articulation Matrix of the IT program at FCITR 
Taleb A. and al. [12] and Al-Mubaid and al. [1] decomposed the Students Outcomes into more detailed elements they called Performance Indicators (PI). For SOs assessment needs, only selected questions are mapped to PIs and embedded in different assessment tools such as exams, homework, projects and assignments. Sundararajan [10] used the Course Learning Outcomes (CLOs) as a primary artifact for SOs assessment, while Taleb A. and al. [12] used them as a semester-based assessment. For each course, the CLOs are mapped to the SOs. Faculty members are asked to select activity/evaluative components and map them to CLOs. Since CLOs are already mapped to SOs, the activity/evaluative components are then mapped to the correspondent SO. However, the level of details in the SOs decomposition (number of PIs or CLOs) is relatively high and makes the assessment process difficult.

Our approach proceeds to a direct decomposition of each SO to a limited set (two or three) of SO Elements (SOEs). It differs also from the SO decomposition work undertaken under the EC-2000 initiative for Engineering programs [2] by the level of decomposition. The latter decomposition is deeply detailed at a certain level that let it be difficult to follow up their assessment and use them as major criteria for evaluation and improvement. Our work is distinguished from the previous research works by the fact that we are considering all the course-related direct/indirect assessments that are undertaken during the semester. All the assessment questions are mapped to the appropriate SOEs using the Bloom's measurable verbs. These assessment questions are going to be evaluated by the faculty members irrespective of the SOs assessment needs, which reduces the workload on faculty members. The web-based system that will support our approach will help in the input of the assessment data and the evaluation processes. We believe that it will contribute considerably to shorten the continuous improvement cycle.

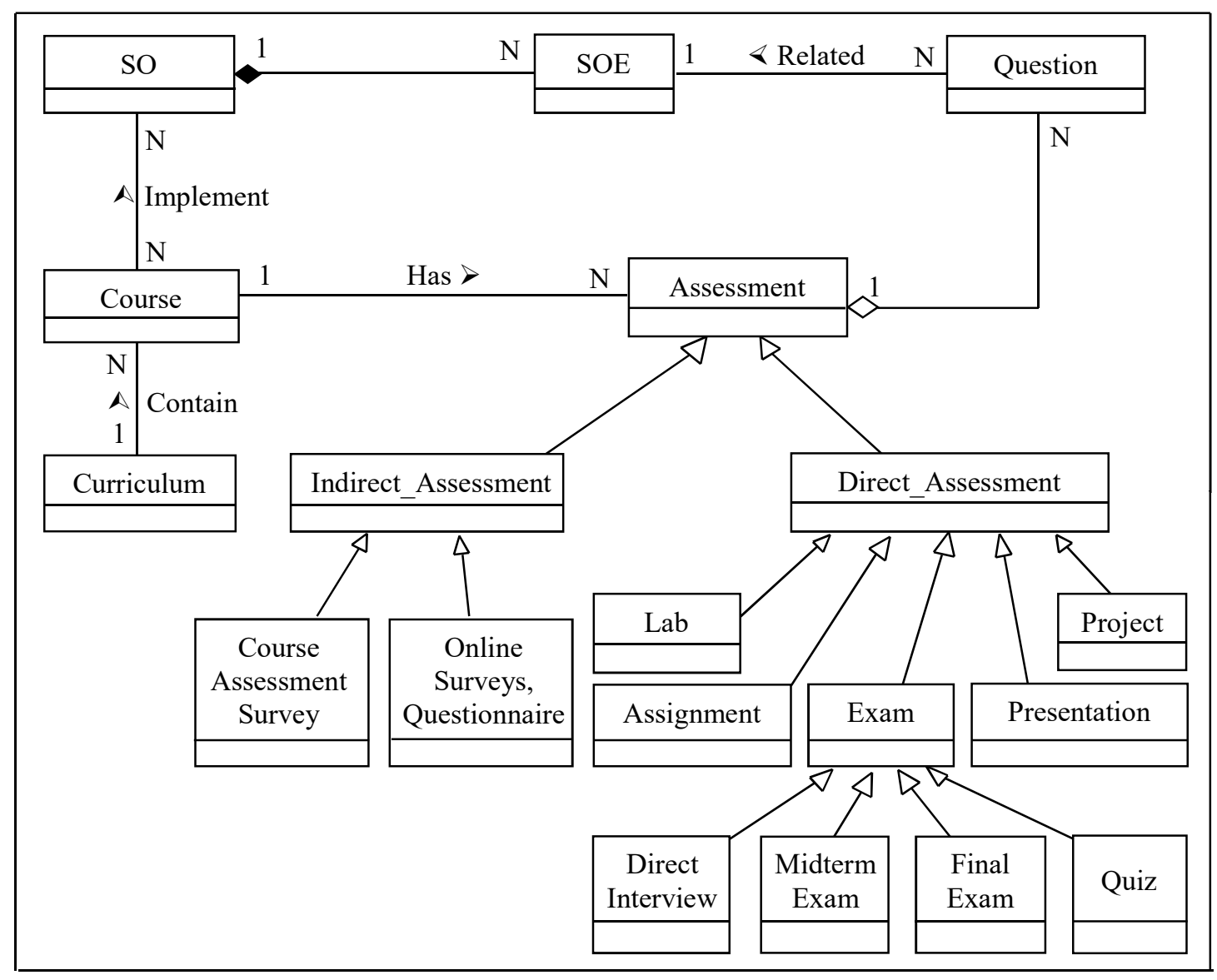

Figure 3-The Class Diagram of the supporting system

The class diagram (Figure 3) describes the main constituents of the supporting system. It shows that a curriculum contains a set of courses. Each course implements a set of SOs that are composed of SOEs. A course has many assessments per semester. Each assessment is composed of a set of questions that should be 
mapped to the appropriate SOE. The assessments are either direct or indirect. Direct assessments may be exams, assignments, presentations, projects or labs. While indirect assessments are course assessment surveys and online surveys/questionnaires.

2. Methodology: The following sections describe the six steps of our methodology. The first three steps can be considered as an inception phase that can be revised during the continuous improvement process. The fourth step corresponds to the assessments implementation and data collection. The fifth step is undertaken at the end of each semester to input the assessments data. The final step proceeds to the analysis of the assessments data and the generation of a set of polar diagrams and bar charts that can be used for the continuous improvement process.

Step\#1- Prepare the articulation matrix using SOs

Each course of the IT curriculum targets or implements a set of SOs. In practice, most of the IT courses implements two to four SOs (except senior projects). The faculty member(s) of each course were responsible for deciding about the SOs that are covered by the course. The final shape of the articulation matrix (Figure 2) was discussed and approved in a department council meeting.

Step \#2- Decompose the SOs into SOEs

Having a deep level of details while decomposing an SO into SOEs makes it difficult to map the SO assessment over the different courses of the curriculum. The detailed articulation matrix (Figure 5) becomes very large; and the assessment process becomes complicated and requires a huge effort. For these reasons, we decided to decompose each SO to a limited set of SOEs (two or three SOEs). Each SO is then examined and decomposed into SOEs that reflects the main different knowledge and skills embodied by the SO. While undertaking these decompositions, we faced two different situations:

a) The $\mathrm{SO}$ contains an enumerated list of knowledge/skills, such as $\mathrm{SO}(\mathrm{c})-$ ability to design, implement, and evaluate... In this situation, the decomposition is based on these identified knowledge/skills (See SOEs(c1, c2 and c3 in Figure 4).

b) The $\mathrm{SO}$ is general and can be even considered as one $\mathrm{SOE}$, such as $\mathrm{SO}(\mathrm{d})$ - ability to function effectively on teams to accomplish a common goal. In this situation, we called for the state of the art and literature to proceeded to a decomposition that covers the main knowledge/skills of the SO (See SOEs(d1, d2 and d3)).

a) An ability to apply knowledge of computing and mathematics appropriate to the discipline

a1: An ability to apply knowledge of computing appropriate to the discipline. (Applying)

a2: An ability to apply knowledge of mathematics appropriate to the discipline. (Applying)

b) An ability to analyze a problem, and identify and define the computing requirements appropriate to its solution

b1: An ability to analyze a problem, and identify the computing requirements appropriate to its solution (Analyzing)

b2: An ability to analyze a problem, and define the computing requirements appropriate to its solution (Analyzing)

c) An ability to design, implement, and evaluate a computer-based system, process, component, or program to meet desired needs

c1: An ability to design a computer-based system, process, component, or program to meet desired needs. (Creating)

c2: An ability to implement a computer-based system, process, component, or program to meet desired needs. (Applying)

c3: An ability to evaluate a computer-based system, process, component, or program to meet desired needs. (Affective Learning)

d) An ability to function effectively on teams to accomplish a common goal

$\mathrm{d} 1$ : An ability to prepare a work schedule for the assigned task and complete it within the appropriate deadlines. (Applying)

d2: An ability to participate in team meetings with full preparedness for providing useful input. (Affective Learning)

d3: An ability to share ideas among the team and promote good communication among the team members. (Affective Learning)

...

Figure 4-Decomposition of SOs (a) to (d) into SOEs 


\section{Step \#3- Prepare the articulation matrix using SOEs}

The articulation matrix that have been prepared in step\#1 is enhanced by the details of step\#2 as shown in the Figure 5 . However, a particular SO that have been previously targeted by a course can now be partially targeted by the same course. For example, the SO (a) includes (a1) and (a2). (a1) is related to the ability to apply knowledge of computing appropriate to the discipline while (a2) is related to the ability to apply knowledge of mathematics appropriate to the discipline. Some courses may implement (a1) but not (a2) and vice-versa. In our case, COIT 260 and COIT 321 implement (a1) but not (a2). However, we paid attention that all SOEs of a particular SO are implemented by at least one course of the curriculum.

\begin{tabular}{|c|c|c|c|c|c|c|c|c|c|c|c|}
\hline \multirow{2}{*}{$\begin{array}{r}\text { SOs }> \\
\text { Courses } \vee\end{array}$} & \multicolumn{2}{|c|}{ (a) } & \multicolumn{2}{|c|}{ (b) } & \multicolumn{3}{|c|}{ (c) } & \multicolumn{3}{|c|}{ (d) } & \multirow[b]{2}{*}{$\cdots$} \\
\hline & (a1) & (a2) & (b1) & (b2) & (c1) & (c2) & (c3) & (d1) & (d2) & (d3) & \\
\hline COIT 260 & $\checkmark$ & & $\checkmark$ & $\checkmark$ & & & & & & & $\ldots$ \\
\hline COIT 321 & $\checkmark$ & & $\checkmark$ & $\checkmark$ & & & & & & & . \\
\hline & & & & & & & & & & & \\
\hline COIT 499 & $\checkmark$ & $\checkmark$ & & & $\checkmark$ & $\checkmark$ & $\checkmark$ & $\checkmark$ & $\checkmark$ & $\checkmark$ & \\
\hline
\end{tabular}

Figure 5 - The detailed articulation matrix with SOEs

\section{Step \#4- Map the assessment questions to SOEs using the Bloom's measurable verbs}

All assessment questions, either direct or indirect, should be mapped to the appropriate SOE. In practice, many problems were faced during the mapping process. These problems are related to ambiguities between some SOs such as "SO(b)-ability to analyze a problem, and identify and define the computing requirements appropriate to its solution" versus "SO(k)-ability to identify and analyze user needs and take them into account in the selection, creation, evaluation and administration of computer-based systems". The ambiguity comes from the fact that user's needs can be considered as a problem to be analyzed. For some courses related to analysis, design and implementation of systems, the ambiguities may be caused by the relationship that may exist between some SOs (b, c, i, k) with the Software Life Cycle (SLC) activities (Analysis, Design, Implementation, etc). In fact, a problem may be related to design or implementation not necessary to the Analysis phase of the SLC.

Benjamin S. Bloom and al. designed a taxonomy that classifies the human thinking into six cognitive levels of complexity [4]. The taxonomy, that has been updated in 2001 [6] [3], includes the following six cognitive levels: Remembering, understanding, applying, analyzing, evaluating and creating. The importance of this taxonomy is that it can be used "as a method of classifying educational objectives, educational experiences, learning processes, and evaluation questions and problems" [9]. In order to help faculty members in their mapping tasks of assessment questions to appropriate SOE, we called for the use of the Bloom's taxonomy to categorize these SOEs into one of the cognitive levels. For each of these levels, a set of measurable verbs are suggested [13] that can be used while writing assessment questions. Using these verbs helps faculty members write questions that are more suitable for a particular SOE.

In FCITR, we studied our SOEs and classified them into one of the Bloom's cognitive levels as shown in the Figure 4. The Bloom's measurable verbs helped our faculty members to write assessment questions that are related to the selected cognitive level and appropriate to SOE.

Step \#5- Input the assessment results for each course's assessments.

Having mapped the assessment questions to the appropriate SOEs, each assessment has a cover page that summarizes the total marks for each SOE. Figure 6 shows a sample of a summary table that is part of the assessment cover page. It shows all course's SOs and the detailed SOEs that are targeted by the assessment. This table is used to record the subtotal for each SOE and the assessment total marks. At the end of the semester, faculty members calculate the total grades they got for each SOE from the different direct assessments. The same work is undertaken separately for the indirect assessments. The web-based supporting system helps faculty members to input the

\begin{tabular}{|c|c|c|}
\hline \multicolumn{3}{|c|}{ Marks } \\
\hline \multirow{2}{*}{$\begin{array}{c}\text { Outcome B } \\
\text { (9 Marks) }\end{array}$} & B1 & $1-$ \\
\hline & B2 & $/ 09$ \\
\hline \multirow{3}{*}{$\begin{array}{l}\text { Outcome C } \\
\text { (4 Marks) }\end{array}$} & C1 & 1 \\
\hline & $\mathrm{C} 2$ & $/ 02$ \\
\hline & $\mathrm{C} 3$ & $/ 02$ \\
\hline \multirow{3}{*}{$\begin{array}{l}\text { Outcome I } \\
\text { (7 Marks) }\end{array}$} & I1 & $/ 04$ \\
\hline & I2 & $/ 03$ \\
\hline & I3 & $1-$ \\
\hline \multirow{2}{*}{$\begin{array}{c}\text { Outcome K } \\
\text { (- Marks) }\end{array}$} & K1 & $1-$ \\
\hline & K2 & $1--$ \\
\hline \multicolumn{2}{|c|}{$\begin{array}{c}\text { Total Marks } \\
\text { (In Figure) }\end{array}$} & /20 Marks \\
\hline \multicolumn{2}{|c|}{$\begin{array}{c}\text { Total Marks } \\
\text { (In Words) }\end{array}$} & \\
\hline
\end{tabular}

Figure 6-A sample exam paper cover page with the correspondent SOEs 
direct and indirect total marks for each SOE.

These data will be summarized at the course level and many outputs will be provided to help faculty members in the preparation of their course file. Other outputs will be provided at the program level to give the overall results related to all IT courses and all SOs.

\section{Step \#6- Analyze the assessment data}

The web-based supporting system provides analysis and help at two different levels: Course level and Program level. At the course level both SOEs and SOs are considered. However, for the program level, only general SOs will be considered.

\section{a) Course level analysis of the assessment data}

At the course level, the system calculates the total marks for each SO, for both the direct and indirect results. Polar diagrams, that are required for the course file, will be provided for each kind of assessment (direct and indirect). Semester after semester, the SOs results can be compared to highlight any improvement.

Figure 7 shows a polar diagram for direct assessments in course COIT 415. The applicable SOs for this course are (b), (c), (i) and (k). The corresponding results for these SOs are respectively $73 \%, 85 \%, 77 \%$ and $69 \%$.

The target success criteria for SO achievement were defined as follows: A student outcome is achieved if students get a $60 \%$ score in the outcome; otherwise, it is not achieved. If the students results are above $80 \%$ they are considered very good. In our case (COIT 415), SOs (b), (i) and (k) are fairly achieved, while SO (c) results are very good. However, there is room left for improvement.

Further analysis of the data entered by faculty members can reveal additional information such as:

- The SOEs that were not addressed by any course assessment. By referring to the detailed articulation matrix we can know if the SOE was targeted by the course or not. If so, the absence of assessments that covers the SOE can be considered as shortcoming.

- The SOEs that were weakly assessed. This can be the case if the SOE got a total marks below a certain threshold decided by the department (less than 5 marks for example).

- The SOEs that were not satisfactory achieved (\% of achievement less than or equal to a given threshold). These SOEs can be subject to improvement in the following semester.

\section{b) Program level analysis of the assessment data}

At the program level, the supporting system calculates the average of each SO for all IT courses, for both direct and indirect results. Two kinds of polar diagrams can be generated: SO-wise and program-wise polar diagrams. The SO-wise polar diagrams (Figure 8) summarizes the students achievements in IT courses that addresses a particular SO. They are useful to highlight in which course an outcome is best or bad achieved. The program-wise polar diagram (Figure 9) shows the students achievements in all SOs.

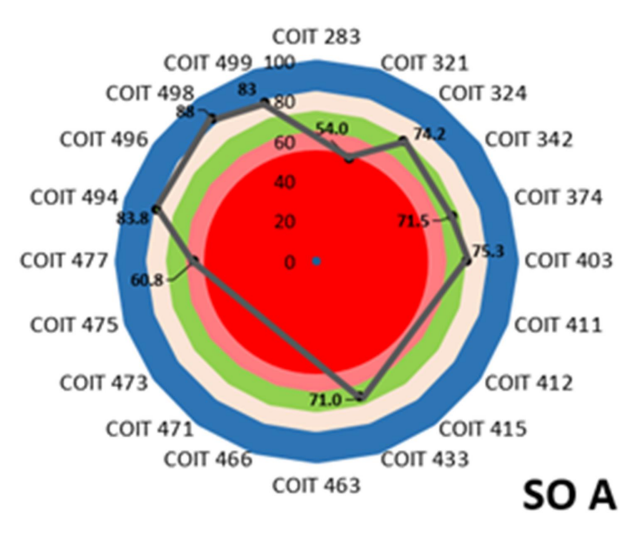

Figure 8-Direct Assessments of SO (A) (Fall, 2017/2018)

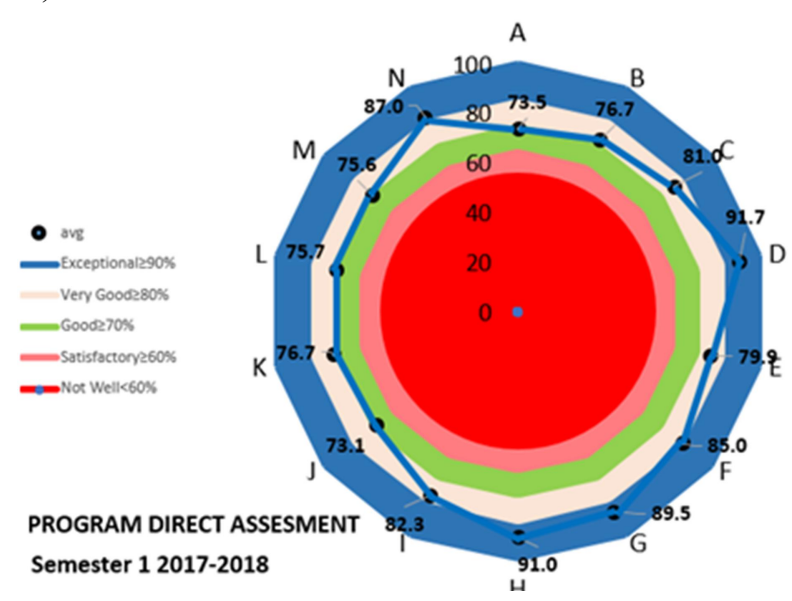

Figure 9-Direct Assessments of the IT Courses (Fall, 2017/2018) 
However, additional polar diagrams or bar charts can be generated to compare the students achievements in two selected semesters (usually two consecutive semesters), either SO-wise or programwise. Figure 10 shows a bar chart giving a program-wise comparison between two consecutive semesters.

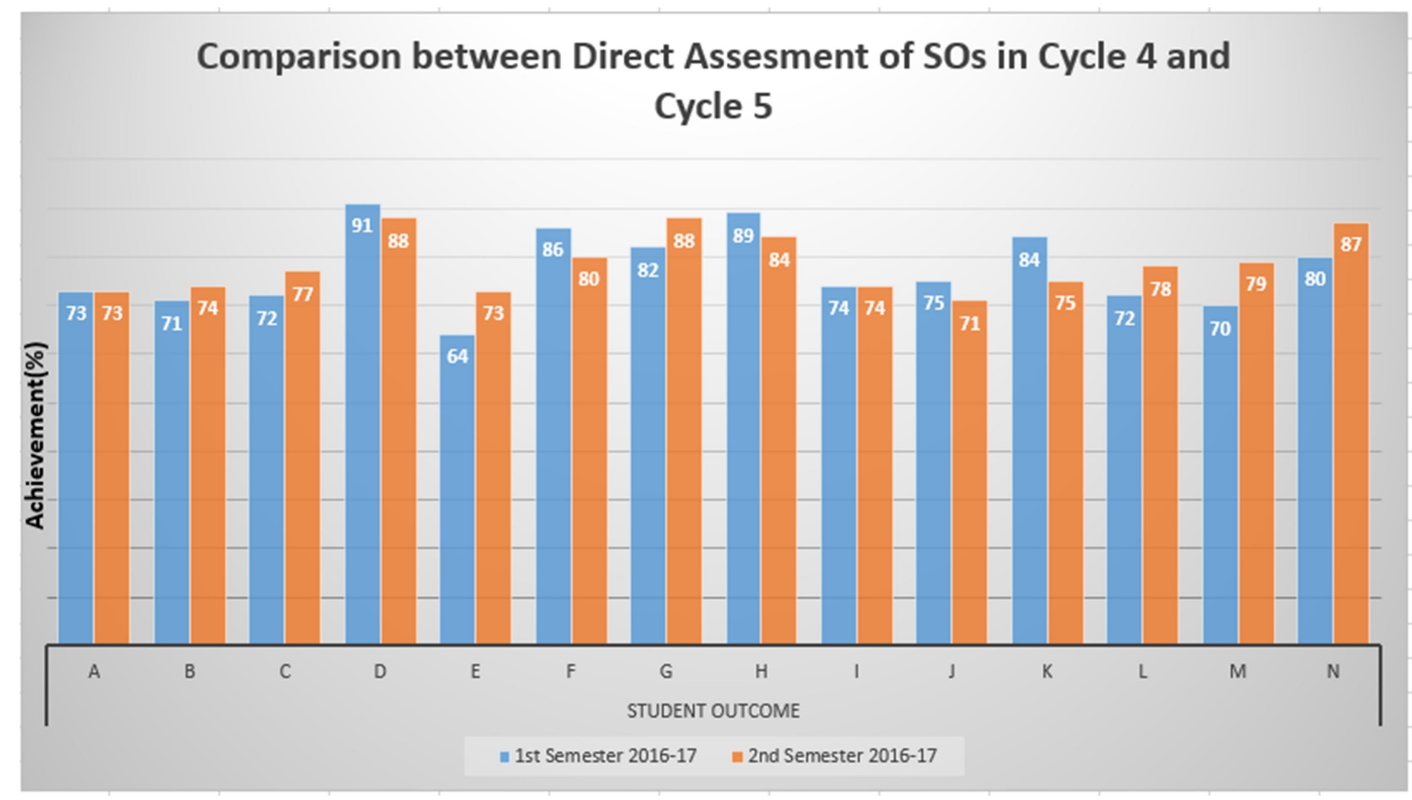

Figure 10-Comparison between direct assessments of the 1st and 2nd semesters 2016/2017

3. Conclusion and future work: In this paper, we presented a six steps methodology that helps in a better implementation and assessment of the Students Outcomes (SOs). Step\#1 prepares an articulation matrix that maps the SOs to the different courses of the IT program. Step\#2 proceeds to the decomposition of the SOs into its elementary contents called SOEs. These SOEs correspond to the main knowledge and skills embodied by the SO. Their identification is a key success to the better implementation of the SOs. Assessing SOs as "all-in-one" is in fact inaccurate and may lead to a poor achievement of the SOs. Using the SOEs we prepare the detailed articulation matrix in step\#3. Each IT course is then mapped to the detailed SOEs and the new matrix is more accurate since some SOEs are no more addressed by some courses. Step\#4 proceeds to the mapping of the assessment questions to the appropriate SOEs. For this matter, we called for the use of the Bloom's taxonomy to categorize each SOE to one of the cognitive levels and make use of the Bloom's measurable verbs to write questions that are more suitable for a given SOE. Step $\# 5$ occurs at the end of each semester to input the assessment data. A web-based system is planned to be used for this purpose to help also in the analysis and improvement processes. The final step\#6, analyzes the inputted data and produces a set of summaries and outputs that are helpful at the course and program levels. As future work, we are planning to implement the described web-based supporting system and use it for the analysis and continuous improvement of the SOs. While many institutions may proceed to the evaluation and improvement using a two-to-four years cycle [7], the use of such system allow the institution to considerably shorten the improvement cycle.

\section{REFERENCES}

[1] Al-Mubaid, H., Kim, D. J., Yue, K. B., Hall, S., \& Abeysekera, K. (2010). Assessment Model and Practices for Computing and Information Systems Programs. In Information Systems Educators Conference ISECON 2010 (Vol. 27, p. 1336).

[2] Besterfield-Sacre, M., Shuman, L. J., Wolfe, H., Atman, C. J., McGourty, J., Miller, R. L., ... \& Rogers, G. M. (2000). Defining the outcomes: A framework for EC-2000. IEEE Transactions on education, 43(2), 100-110. 
[3] Bhakti, S. L. O. W. (2010). Bloom's Taxonomy: Original and Revised

[4] Bloom, B.S. (Ed.), Engelhart, M.D., Furst, E.J., Hill, W.H., \& Krathwohl, D.R. (1956). Taxonomy of educational objectives: The classification of educational goals. Handbook 1: Cognitive domain. New York: David McKay.

[5] Criteria for accrediting computing programs Effective for Reviews During the 2017-2018 Accreditation Cycle. ABET, Inc. (accreditation@abet.org) Copyright 2005. http://www.abet.org/wp-content/uploads/2016/12/C001-17-18-CAC-Criteria-10-29-16-1.pdf

[6] David R. Krathwohl. (2002). A Revision of Bloom's Taxonomy:An Overview. THEORY INTO PRACTICE, Volume 41, Number 4, Autumn 2002, Copyright (C) 2002 College of Education, The Ohio State University.

[7] Gloria M. Rogers.(2005). Establishing Timelines and Responsibilities-An Example. ABET, Inc. (grogers@abet.org)Copyright 2005. http://www.abet.org/wp-content/uploads/2015/04/establish-assess-timelines-responsibilities-2.pdf

[8] G. Rogers, "Direct and Indirect Assessments: What Are They Good For?", Assessment 101, Assessment Tips with Gloria Rogers, Community Matters, A Monthly Newsletter for the ABET Community, August 2006, p.3.

[9] Richard, P. (1985). Bloom's taxonomy and critical thinking instruction. Educational leadership, 42(8), pp.36.

[10] Sundararajan, S. (2014). A strategy for sustainable student outcomes assessment for a Mechanical Engineering program that maximizes faculty engagement. In Mechanical Engineering Conference Presentations, Papers, and Proceedings.

[11] Shryock, K., \& Reed, H. (2009, June). ABET accreditation: Best practices for assessment. In Proceedings of the 2008 ASEE Gulf-Southwest Annual Conference, the University of New Mexico, Albuquerque.

[12] Taleb A., Al-Shargabi M., Taleb N. (2016). Multi-Direct Assessment Methods for Better Program Improvement . In Proceedings of the 6th International Arab Conference on Quality Assurance in Higher Education (IACQA'2016).

[13] VERBS, C. L. I. Bloom's Taxonomy of Measurable Verbs. Center for Teaching \& Learning| The University of Georgia| ctl. uga. edu, 706, 1355. 